\title{
Azimuthally Polarized, Circular Colloidal Quantum Dot Laser Beam Enabled by a Concentric Grating
}

Yuan Gao, ${ }^{\dagger}$ Landobasa Y. M. Tobing, ${ }^{\dagger}$ Aurélien Kiffer, ${ }^{\dagger}$ Dao Hua Zhang, ${ }^{\dagger}$ Cuong Dang, ${ }^{*},+\ddagger$ and Hilmi Volkan Demir* ${ }^{\dagger} \dagger, \S, \#$

${ }^{\dagger}$ LUMINOUS! Center of Excellence for Semiconductor Lighting and Displays, Satellite Research Centre, Centre for Optoelectronics and Biophotonics, The Photonics Institute, School of Electrical and Electronic Engineering and The Photonics Institute, Nanyang Technological University, 50 Nanyang Avenue, 639798, Singapore

${ }^{\ddagger}$ CNRS International NTU Thales Research Alliance (CINTRA), Research Techno Plaza, 50 Nanyang Drive, Border X Block, 637553, Singapore

${ }^{\S}$ Division of Physics and Applied Physics, School of Physical and Mathematical Sciences, Nanyang Technological University, 21 Nanyang Link, 637371, Singapore

${ }^{\#}$ Department of Electrical and Electronics Engineering and Department of Physics, UNAM-Institute of Materials Science and Nanotechnology, Bilkent University, Bilkent, Ankara, Turkey

\section{Supporting Information}

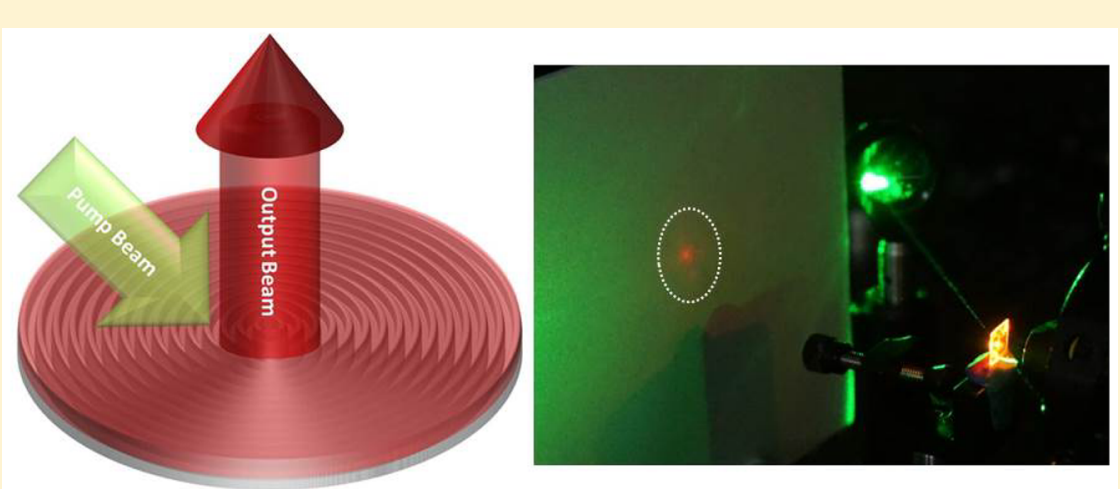

ABSTRACT: Since optical gain was observed from colloidal quantum dots (CQDs), research on CQD lasing has been focused on the CQDs themselves as gain materials and their coupling with optical resonators. Combining the advantages of a CQD gain medium and optical microcavity in a laser device is desirable. Here, we show concentric circular Bragg gratings intimately incorporating $\mathrm{CdSe} / \mathrm{CdZnS} / \mathrm{ZnS}$ gradient shell CQDs. Because of the strong circularly symmetric optical confinement in two dimensions, the output beam CQD-based circular grating distributed feedback laser is found to be highly spatially coherent and azimuthally polarized with a donut-like cross section. We also observe the strong modification of the photoluminescence spectrum by the grating structures, which is associated with modification of optical density of states. This effect confirmed the high quality of the resonator that we fabricated and the spectral overlap between the optical transitions of the emitter and resonance of the cavity. Single mode lasing has been achieved under a quasi-continuous pumping regime, while the position of the lasing mode can be conveniently tuned via adjusting the thickness of the CQD layer. Moreover, a unidirectional output beam can be observed as a bright circular spot on a screen without any collimation optics, presenting a direct proof of its high spatial coherence.

KEYWORDS: colloidal quantum dot, lasing, circular grating, azimuthal polarization, DFB

olloidal quantum dots (CQDs), which are regarded as "nanoscience building blocks", 1 have attracted a lot of research interest globally for more than three decades. ${ }^{2}$ One of the most intriguing properties of semiconductor CQDs is that their electronic structures are tunable flexibly via adjusting their size, chemical composition, morphology, or adopting heterostructures. ${ }^{3}$ As a result, the light emission from the semiconductor CQDs can be designed to cover the entire visible spectral range with a high quantum yield and a narrow line width. Moreover, considering their tunable emission wavelength, CQDs are able to bridge the so-called "green gap" in conventional light emitters. ${ }^{4}$ Given their unique optical properties, CQDs are very promising in improving the qualities of modern lighting and displays.

Due to the so-called quantum confinement effect, the semiconductor CQDs exhibit discrete energy levels, and such discrete energy levels make them applicable to fabrication of thermally stable lasers. ${ }^{7,8}$ After Klimov and his colleagues realized CdSe

Received: September 18, 2016

Published: November 7, 2016 
CQD lasing at room temperature in 2000, the research on CQD lasing has been rising. ${ }^{7,9}$ Single-exciton gain in CQDs has been shown to overcome the Auger process, which is a fundamental problem of CQD lasing. ${ }^{10,11}$ Red, green, or blue (RGB) lasing from semiconductor CQDs has been demonstrated by incorporation with various optical feedback configurations, such as Fabry-Pérot cavities, ${ }^{12,13}$ whispering gallery mode (WGM), ${ }^{14-16}$ distributed feedback (DFB) lasers, ${ }^{17-19}$ and others. ${ }^{20}$ However, most of the works did not show the output laser beam from the laser devices, which is direct evidence of the spatial coherence of lasers. ${ }^{21}$ In this work, we show the first account of a highly spatially coherent CQD-based surface-emitting circular grating DFB (CG-DFB) laser.

A conventional one-dimensional (1D) DFB laser can provide optical feedback in only one direction. Therefore, the surface outcoupling beam has different divergences in the orientations along and normal to the gratings. This problem, in essence, can be addressed by fabricating the gratings in a circular fashion. ${ }^{22}$ Because of the strong and in-plane symmetric confinement of the resonant mode, the CG-DFB lasers exhibit a low operation threshold and a low-divergent circularly symmetric output beam. $^{23,24}$ Moreover, compared with conventional verticalcavity-surface-emitting lasers (VCSELs), CG-DFB lasers are expected to possess a high output power due to their large in-plane gain area. ${ }^{24}$ Thus, the combination of two-dimensional light confinement in CG-DFB and the optical properties of CQDs would make the CQD-based CG-DFB laser an excellent candidate for achieving high-power single-mode lasers with high $Q$ factor at any desired wavelength within the visible spectrum.

In this paper, we report the first account of CQD-based CG-DFB lasers. A strong modification of the optical density of states (DOS) of the CQDs that were coupled with the circular grating was observed, which is confirmed by two proofs. First, a dent, which was associated with the position of the photonic stop band, appeared in the corresponding fluorescence spectrum. Second, we showed a prolonged lifetime of the transitions that lie within the stop band. As the sample is optically pumped, a singlemode lasing peak emerges at the edge of the stop band, where the optical DOS is enhanced. By characterizing the output lasing, we confirmed that the lasing beam is azimuthally polarized and highly directional, which is a direct proof of the spatial coherence of our CQD-based CG-DFB laser. The findings indicate that the proposed microlaser based on the intimate integration of CQDs into a circular Bragg grating is very promising for various photonic applications that require surface normal geometry and good beam quality.

The fabrication of a circular Bragg substrate, formed by 250 concentric circular grooves with a $376 \mathrm{~nm}$ pitch, was carried out by electron beam (e-beam) lithography followed by dry etching. The positive-tone ZEP520A resist was first spin-coated on a $0.5 \mathrm{~mm}$ thick quartz substrate at $150 \mathrm{~nm}$ thickness, followed by a $30 \mathrm{~nm}$ conductive polymer film (ESPACER) for avoiding charging effects during e-beam patterning. The e-beam exposure was carried out at a $65 \mu \mathrm{C} / \mathrm{cm}^{2}$ dose range, and the sample was developed in methyl isobutyl ketone (MIBK) at $6{ }^{\circ} \mathrm{C}$ for $10 \mathrm{~s}$. The dry etching process was based on $30 \mathrm{sccm} \mathrm{CF}_{4}$ and $30 \mathrm{sccm}$ $\mathrm{CHF}_{3}$ gases at $150 \mathrm{mT}$ pressure, using $170 \mathrm{~W}$ radio frequency (RF) power for $555 \mathrm{~s}$. Then, the resist was removed with piranha solution (a mixture of concentrated $\mathrm{H}_{2} \mathrm{SO}_{4}$ and $\mathrm{H}_{2} \mathrm{O}_{2}$ with a ratio of 3:1). The period of the circular grating is $376 \mathrm{~nm}$. The scanning electron microscopic (SEM) images of the substrate are presented in Figure 1. A thin layer of poly(3,4-ethylenedioxythiophene) polystyrenesulfonate (PEDOT:PSS) was temporally spin-coated
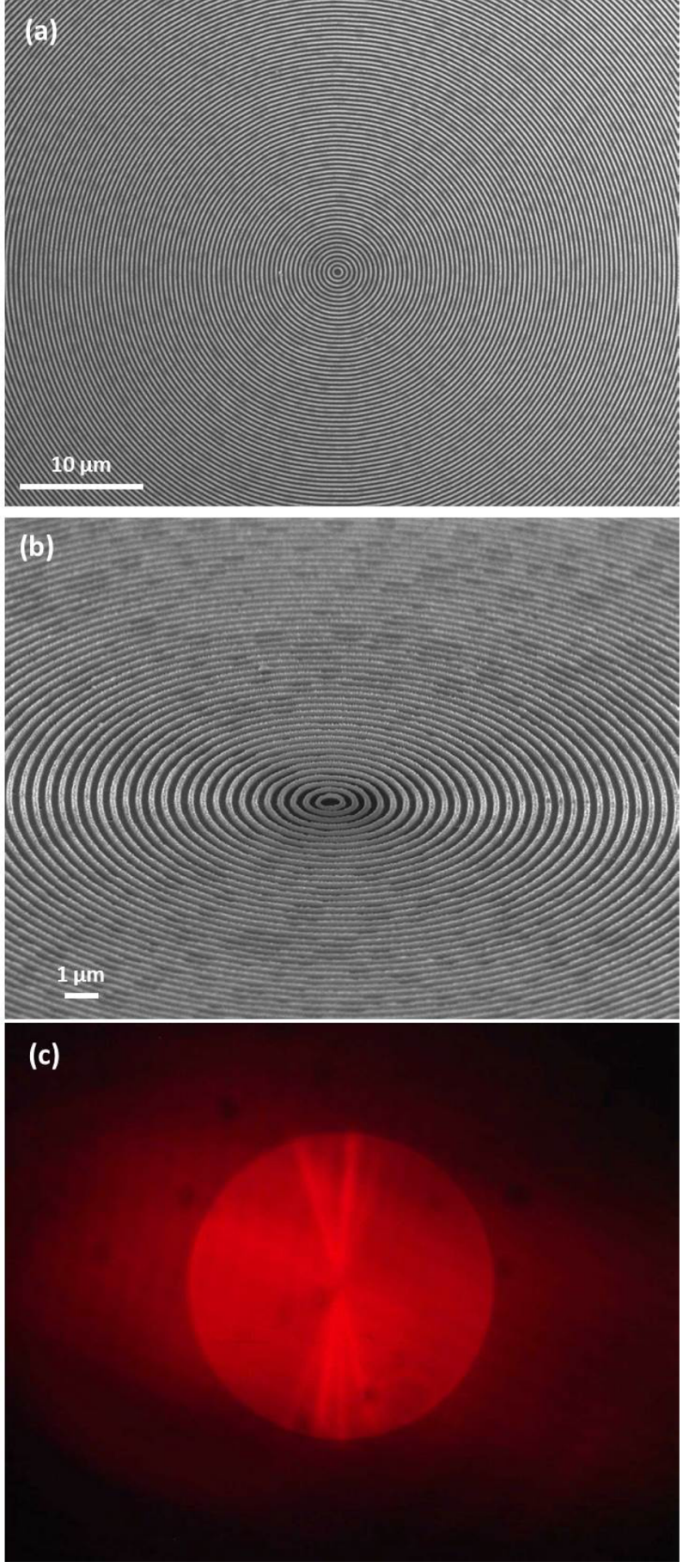

Figure 1. SEM images of the circular Bragg grating (a thin layer of PEDOT:PSS was spin-coated to avoid charge accumulation) observed at (a) $0^{\circ}$ and (b) $45^{\circ}$. (c) Far-field radiation pattern of the CQDs on the circular grating substrate under low excitation level. The diameter of the circular grating pattern is $188 \mu \mathrm{m}$.

on the quartz substrate surface to increase its electrical conductivity and avoid charging during SEM inspection. As can be seen from the SEM images, the fabricated circular grating was free from defects, and the roughness resulting from the dry etching process is in the deep-subwavelength range, suggesting that the as-fabricated circular Bragg gratings are promising for high-quality laser applications. In addition, the quartz circular Bragg substrate can serve as a mold for imprinting the grating pattern onto other flexible substrates. ${ }^{18,25,26}$ 
(a)

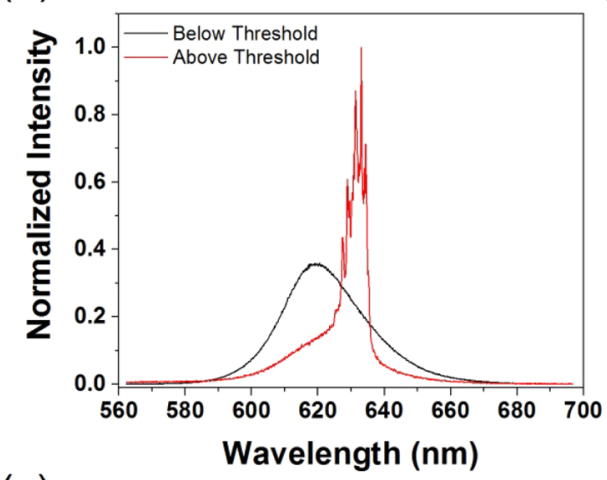

(c)

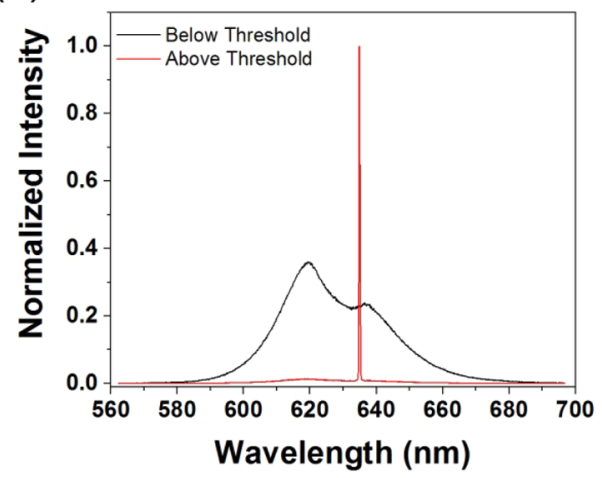

(e)

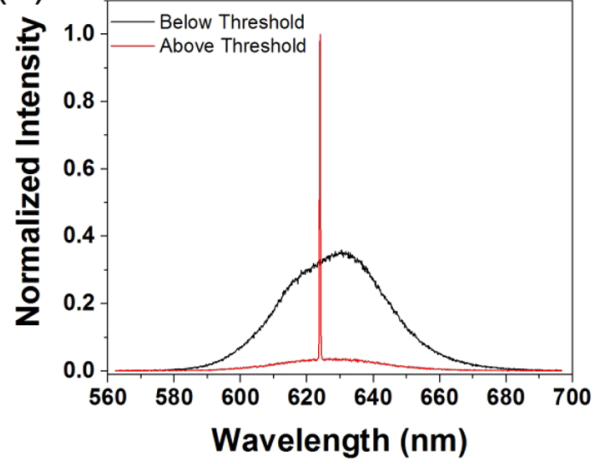

(b)

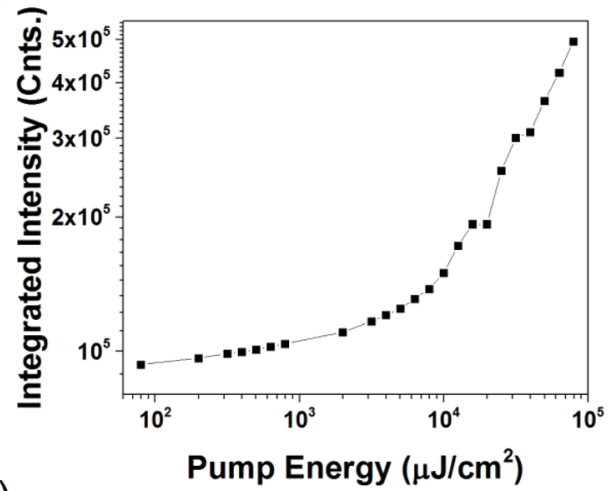

(d)

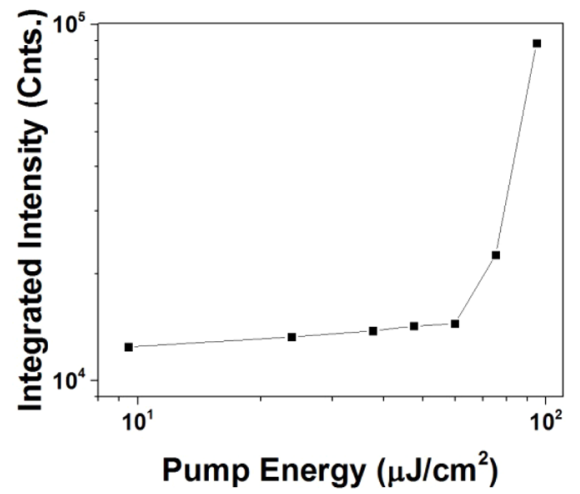

(f)

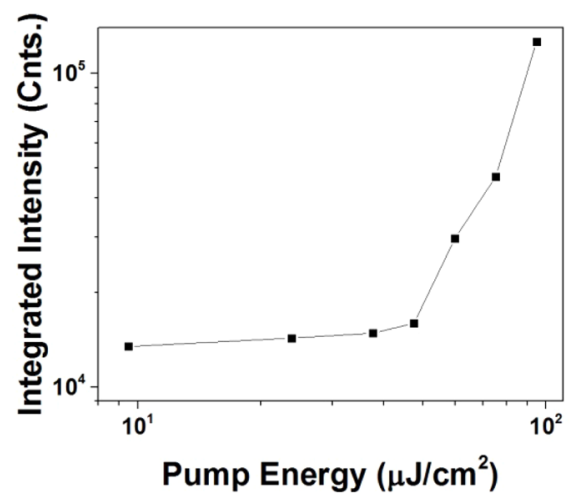

Figure 2. Characterization of the CQDs as gain medium and the CQD-CG-DFB lasers. (a) Normalized emission spectra of CQDs lying outside the circular gratings in the CQD film with a thickness of $247 \mathrm{~nm}$ below and above the ASE threshold. (b) Variation of integrated ASE intensity as a function of the pump energy. Normalized emission spectra of CQDs that coupled with the circular gratings below and above the lasing threshold and the integrated lasing intensity as a function of pump energy in the CQD film with a thickness of $247 \mathrm{~nm}$ (c, d) and $203 \mathrm{~nm}(\mathrm{e}, \mathrm{f})$.

The semiconductor CQDs ( $\mathrm{CdSe} / \mathrm{CdZnS} / \mathrm{ZnS}$ core-shell structures) that were deployed as the gain medium were synthesized with a one-pot method, ${ }^{27}$ as shown in the high-resolution transmission electron microscopy (HRTEM) image in Figure S1a. The $\mathrm{ZnS}$ shells not only increase the absorption cross section of the CQDs but also ensure a good passivation of the surface defects of the cores. Therefore, the photoluminescence (PL) spectrum of the $\mathrm{CdSe} / \mathrm{CdZnS} / \mathrm{ZnS} \mathrm{CQD}$ solution exhibits a narrow line width and Gaussian-like symmetric shape, which can be seen from Figure S1b. Meanwhile, the gradient composition shells and the quasi-type-II band alignment alleviate the Auger effect in such $\mathrm{CdSe} / \mathrm{CdZnS} / \mathrm{ZnS}$ CQDs, which is desirable for achieving low-threshold lasing action in CQDs. ${ }^{28,29}$ The PL quantum yield (PLQY) for the diluted $\mathrm{CdSe} / \mathrm{CdZnS} / \mathrm{ZnS} \mathrm{CQD}$ solution was 0.62 , measured with an integrating sphere and excitation of a $405 \mathrm{~nm}$ laser.

A densely packed and uniform CQD film was prepared by spin-coating a concentrated $\mathrm{CdSe} / \mathrm{CdZnS} / \mathrm{ZnS}$ CQD solution
$(100 \mathrm{mg} / \mathrm{mL})$ onto the quartz substrate at a rate of $1000 \mathrm{rpm} /$ min. The thickness of the CQDs film was $246.52 \pm 6.49 \mathrm{~nm}$, which was characterized with an ellipsometer (VASE VB-250). The sample was pumped at $532 \mathrm{~nm}$ by a frequency-doubled $\mathrm{Nd}$ :YAG laser with a repetition rate of $60 \mathrm{~Hz}$ and pulse width of $0.5 \mathrm{~ns}$. The focused laser spot was adjusted to cover the entire region of a circular grating pattern, and the spot size was about $4.21 \times 10^{-4} \mathrm{~cm}^{2}$. The signals were collected vertically to the sample surface by an objective lens and analyzed with a monochromator (ANDOR Shamrock 303i) and a charge-coupled device (CCD) (ANDOR iDus 401); meanwhile, the excitation spot was monitored with a CCD camera (Thorlabs). As shown in Figure 1c, under low excitation energy, the CQDs that were in the circular grating pattern area are brighter than those that were outside, which is attributable to the increased surface-vertical light scattering from the circular grating. The PL spectrum of the $\mathrm{CdZnSeS} / \mathrm{ZnS}$ CQD film with the excitation spot located 
outside the circular gratings is shown in Figure 2a. The PL spectrum exhibits a $2.2 \mathrm{~nm}$ red shift compared to that of the diluted solution; this is due to an increased photon reabsorption and energy transfer between the CQDs. As the pumping energy was increased, the amplified spontaneous emission (ASE) peak occurred on the red side of the PL peak of the CdSe/CdZnS/ZnS CQDs, as shown in Figure 2a. The ASE peaks range from 624 to $636 \mathrm{~nm}$, and the spiky peaks are results from the random optical feedbacks within the CQDs film (a random laser). The variation of integrated intensity of the ASE peaks as a function of pumping power is plotted in Figure 2b, which shows a so-called "soft threshold" and a slow intensity building up above the threshold. This "soft threshold" is a result of a considerable amount of spontaneous emission collected by the detector ${ }^{30}$ and a small amount of ASE scattered vertically to the detector. When the excitation spot was moved to a circular grating pattern and under low excitation energy, the emission spectrum was suddenly changed, if it is compared with that from CQDs outside the gratings, as shown in Figure $2 \mathrm{c}$. The dent in this spectrum illustrates the position of the stop band. The emission within this stop band was suppressed, because of a reduced optical DOS in the stop band. To confirm the existence of the stop band, the fluorescence lifetime of the $\mathrm{CdSe} / \mathrm{CdZnS} / \mathrm{ZnS} \mathrm{CQDs}$ on the substrate was measured with a confocal scanning fluorescence lifetime imaging (FLIM) system. The sample was excited by a pulsed laser with a wavelength of $375 \mathrm{~nm}$, repetition rate of $20 \mathrm{MHz}$, and pulse duration of $100 \mathrm{ps}$. The lifetime mapping area is around $126 \mu \mathrm{m}$ by $126 \mu \mathrm{m}$, and the lifetime of each pixel within the mapping area has been recorded. The spectral window of the measurement was $626 \pm 10 \mathrm{~nm}$, which corresponded to the stop band of the CG-DFB laser. The distributions of the fluorescence lifetime of CQDs in the lifetime mapping region located within or outside the circular grating pattern are displayed in Figure 3. According to the histograms, the fluorescence lifetime distribution of the CQDs that coupled to the circular grating was longer than those that were outside the grating. This is because the optical transitions, which lie in the stop band, of the emitters that coupled with the grating were suppressed. Therefore, the circular grating strongly modifies the optical DOS of the assembly.

On the other hand, at the edge of the stop band, the optical DOS could be enhanced. ${ }^{31}$ As the CQDs coupled with the circular grating were pumped with a higher energy, a sharp lasing peak $(634.9 \mathrm{~nm})$ appeared at the longer wavelength edge of the stop band. The optical DOS was high at the band edge, resulting in a low group velocity. Therefore, the optical transitions were more favored, and the photons underwent a high optical gain at the stop band edge. ${ }^{32}$ The $Q$ factor of this single-mode lasing, deduced by $\lambda / \delta \lambda$, is at least 2531 (the determination of the $Q$ factor is limited by the resolution, 0.26 at $635 \mathrm{~nm}$, of the spectrometer that we employed). The lasing threshold was 2 orders lower than the ASE threshold of CQDs that were outside the circular grating. This is because the circular Bragg gratings provided a strong two-dimensional confinement in the waveguide and photons that were propagating in the waveguide can be coupled to only one optical mode. Moreover, considering the pulse width $(0.5 \mathrm{~ns})$ of the laser for optical pumping, which is much longer compared to the time of Auger recombination, our CQD-CG-DFB laser can work under a quasi-continuous wave pumping condition. ${ }^{9,19}$ The power efficiency of our demonstrated CQD-based CG-DFB laser is $0.15 \%$. The output intensity was maintained after running continuously for $2 \mathrm{~h}$ under pumping of $183 \mu \mathrm{J} / \mathrm{cm}^{2}$.

The quartz circular Bragg grating substrate is reusable. After the spin-coated layer was rinsed away and the substrate was cleaned thoroughly by immersing in piranha solution, a thinner CQD film with a thickness of $203.18 \pm 2.88 \mathrm{~nm}$ was prepared via spin-coating at a spin rate of $1500 \mathrm{rpm} / \mathrm{min}$. As evident from Figure $2 \mathrm{e}$, the $\mathrm{PL}$ emission of CQDs that lies on the grating differed from that of normal CQDs as well. The position of the modification of the spectrum was blue-shifted to the wavelength of around $620 \mathrm{~nm}$. Given the refractive index of a CQD thin film ( $n=1.87$, determined via an ellipsometer) is significantly higher than that of a quartz grating substrate $(n=1.5)$, the effective refractive index of the laser structure is decreased when the CQD film thickness is reduced. Therefore, the position of the stop band moved to a shorter wavelength. ${ }^{18,19}$ A single-mode lasing peak emerged at $624.9 \mathrm{~nm}$, and the corresponding $Q$ factor is at least 2546. The power transfer function is plotted in Figure $2 \mathrm{f}$, and the lasing threshold is comparable to that of the thick CQDs film. Therefore, the emission wavelength of the CQD-based CG-DFB laser can be readily tuned within the gain profile merely by a simple adjustment of the CQD layer thickness.

The far-field radiation pattern of the CQD-CG-DFB laser was monitored by a microscope and a CCD camera. As shown in Figure $4 \mathrm{a}$, at the very center of the far-field pattern, there was a dim spot. This zero electric field at the center resulted from the cylindrical boundary condition and the azimuthally polarized emission from the CG-DFB laser. ${ }^{26,33,34}$ The polarization of the emission was analyzed by a polarizer before the CCD camera. The variations of the far-field radiation images as a function of the (a)

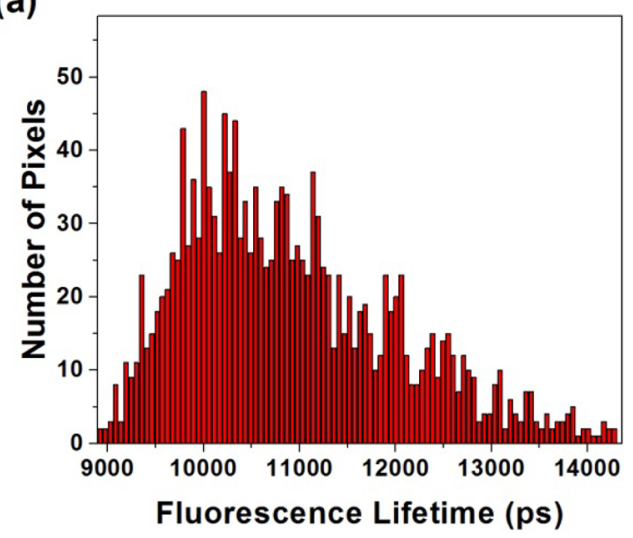

(b)

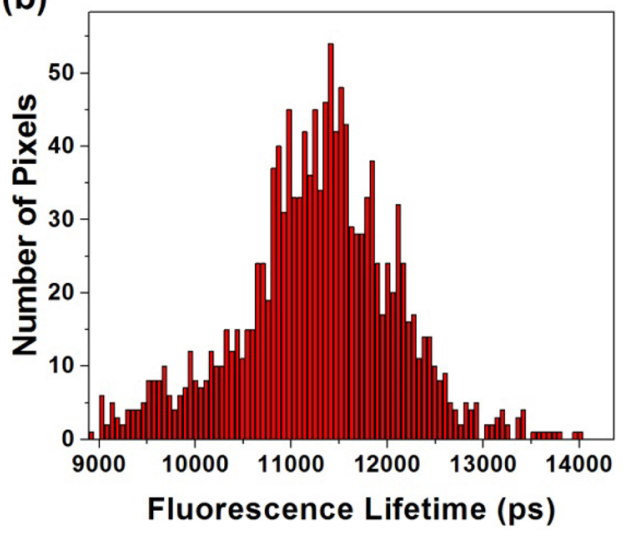

Figure 3. Fluorescence lifetime distribution CQDs in the mapping region that were located outside (a) and within (b) the circular grating pattern. 

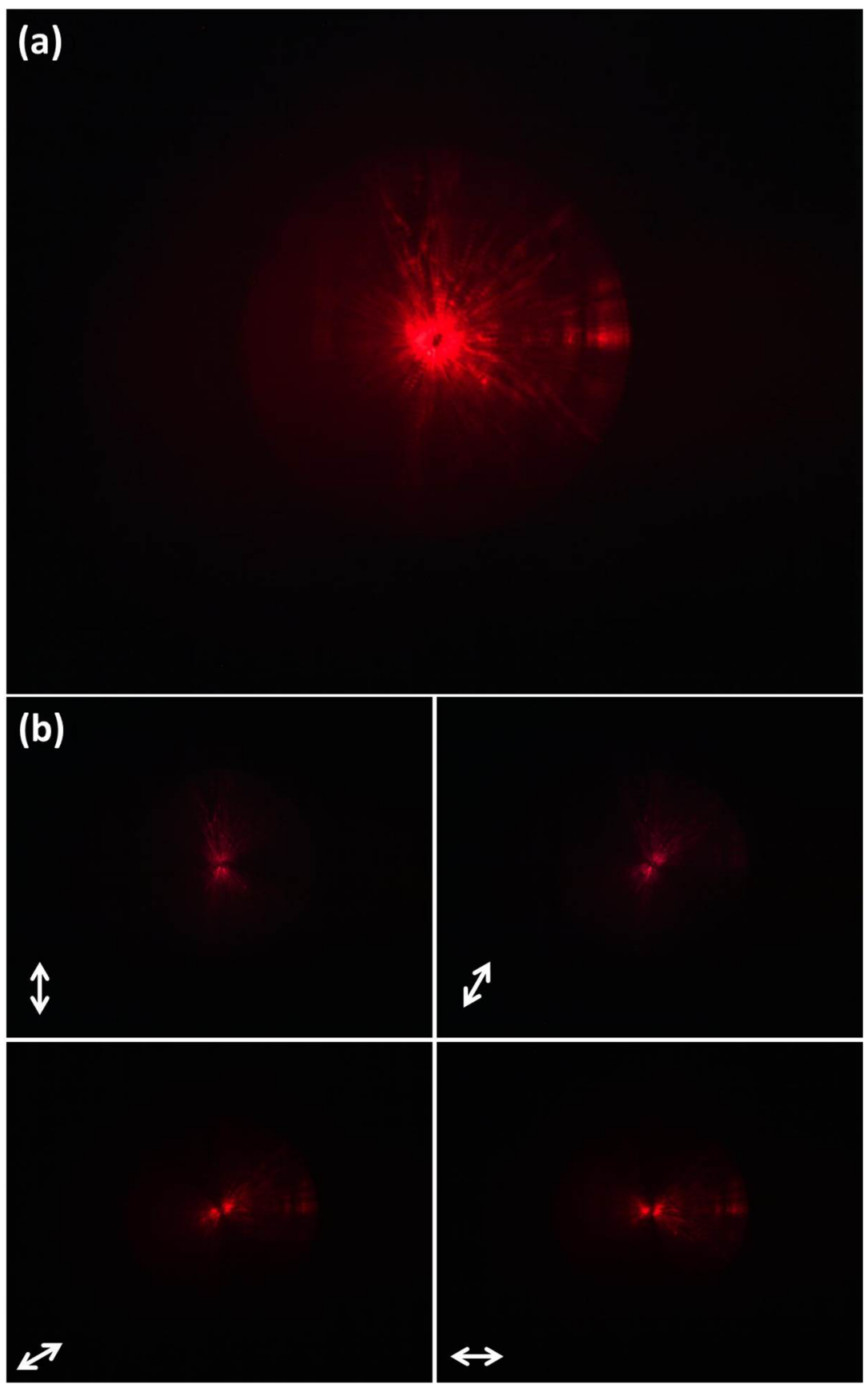

Figure 4. (a) Far-field radiation images that were captured via a CCD camera, when the CQD-CG-DFB laser was operating above the lasing threshold. (b) Variations of the far-field pattern CQD-CG-DFB laser on tuning the axis of the linear polarizer that the laser beam went through.

orientation of the polarizer axis are shown in Figure $4 \mathrm{~b}$, which perfectly illustrates the azimuthally polarized nature of the laser action from the CQD-CG-DFB laser. Thus, both the zero field at the beam center and the polarization-dependent far-field radiation patterns clearly confirm that the out-coupled beam possesses azimuthal polarization.

Although CQD lasing has been reported in various laser schemes, only a few of them demonstrated an outcoupled lasing beam, which directly proves the spatial coherence of a laser. ${ }^{29}$ Due to the circularly symmetric nature of the light confinement, in addition to the light outcoupling via surface-normal secondorder scattering, the outcoupled beam from the CG-DFB has a circular cross section and is expected to be highly unidirectional. In Figure 5a, a viewing screen was placed behind the CQDs-
CG-DFB laser without lens or mirror to focus or collimate the output laser beam. When the CQD-CG-DFB was pumped above its lasing threshold, a bright circular light spot can be seen on the viewing screen (Figure $5 b$ ). This demonstrates that our CQD-CG-DFB laser exhibits an excellent spatial coherence, which is advantageous for a laser scheme to be practical.

In this work, we have demonstrated, for the first time, a semiconductor NC-based CG-DFB laser that has a significant modification on the optical DOS of the semiconductor NC emitters and a highly spatially coherent output beam. We coupled a uniform NC layer with the concentric circular Bragg grating of $376 \mathrm{~nm}$ pitch and observed the modifications of emission spectra of CQDs at the grating region indicating the position of the stop band and the enhanced DOS at the band edge. 


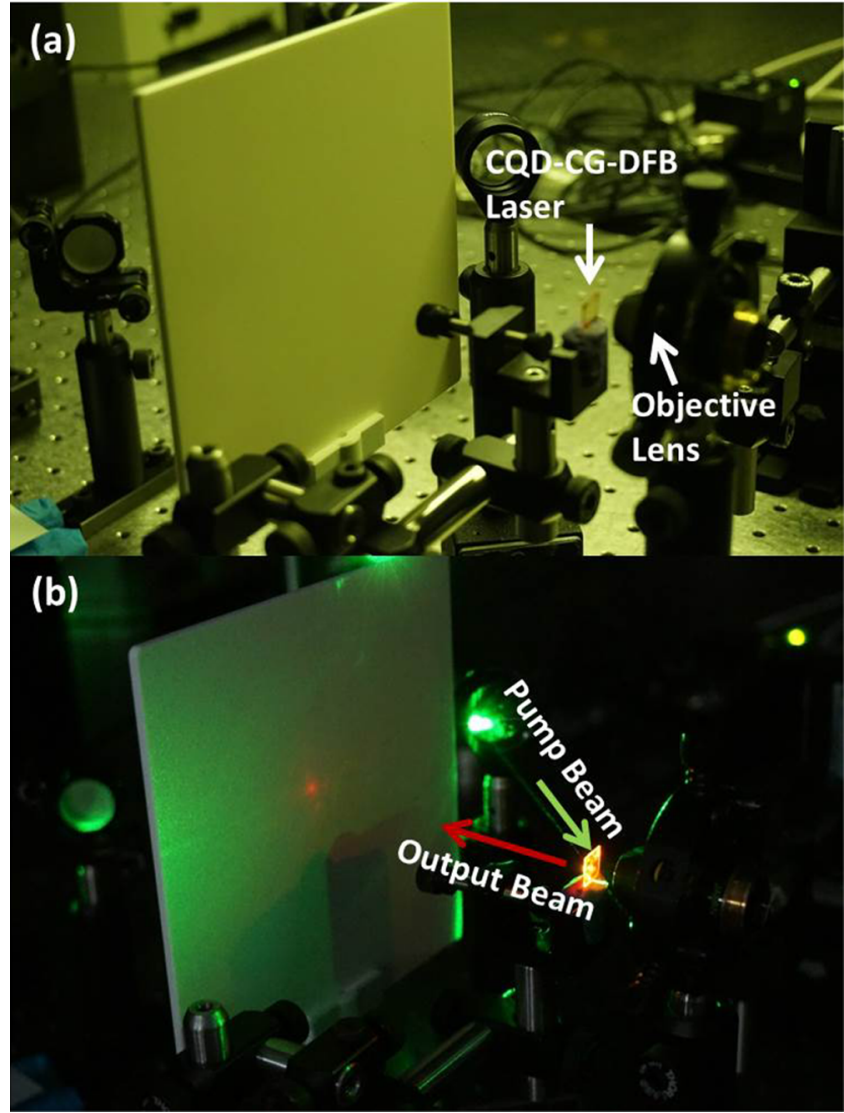

Figure 5. Demonstration of the spatial coherence of the output laser beam from the CQD-CG-DFB device. (a) Image of the measurement setup without optical pumping laser. (b) Image of the output beam when the CQD-CG-DFB laser was optically pumped above the lasing threshold.

At increased pump energy, a sharp single-mode lasing peak emerged at the edge of the stop band. Due to the cylindrical boundary condition that is associated with the circularly symmetric geometry of the CQD-GC-DFB laser, the output beam is azimuthally polarized with zero amplitude at the very center of the beam and at emission direction normal to the device surface. The combination of strong two-dimensional light confinement and single-mode lasing has led to the dramatic reduction of the lasing threshold by 2 orders of magnitude. The high directionality of the laser is also demonstrated by projecting the output beam onto the screen without employing any optical components for focusing or collimating purposes. In light of its surface-normal emission, low lasing threshold, single-mode lasing operation, circular beam cross section, azimuthal polarization, and high spatial coherence, along with the unique optical properties of CQDs, the CQD-CG-DFB laser can find promising applications in various fields such as displays, lighting, photonic circuits, and high-power lasers.

\section{ASSOCIATED CONTENT}

\section{S Supporting Information}

The Supporting Information is available free of charge on the ACS Publications website at DOI: 10.1021/acsphotonics.6b00712.

Additional information (PDF)

\section{AUTHOR INFORMATION}

\section{Corresponding Authors}

*E-mail: hcdang@ntu.edu.sg.

*E-mail: hvdemir@ntu.edu.sg.

\section{ORCID}

Yuan Gao: 0000-0001-9407-1528

Notes

The authors declare no competing financial interest.

\section{ACKNOWLEDGMENTS}

The authors would like to thank the financial support from Singapore National Research Foundation under NRF-NRFI2016-08, the Singapore Agency for Science, Technology and Research (A*STAR) SERC Pharos Program under Grant No. 15273 00025, and NTU start-up grant and AcRF Tier1 grant RG70/15 from Ministry of Education. The authors also acknowledge the financial support from Ministry of Education (RG86/13), Economic Development Board (NRF2013SAS-SRP001-019), and Asian Office of Aerospace Research and Development (FA2386-14-1-0013). The transmission electron microscopy imaging was performed at the Facility for Analysis, Characterization, Testing and Simulation (FACTS) at Nanyang Technological University, Singapore.

\section{REFERENCES}

(1) Alivisatos, A. P. Birth of a Nanoscience Building Block. ACS Nano 2008, 2, 1514-1516.

(2) Rogach, A. Quantum Dots Still Shining Strong 30 Years On. ACS Nano 2014, 8, 6511-6512.

(3) Talapin, D. V.; Lee, J.-S.; Kovalenko, M. V.; Shevchenko, E. V. Prospects of Colloidal Nanocrystals for Electronic and Optoelectronic Applications. Chem. Rev. 2010, 110, 389-458.

(4) Coleman, J. J.; Bryce, A. C.; Jagadish, C.Advances in Semiconductor Lasers; Elsevier/Academic Press, 2012.

(5) Supran, G. J.; Shirasaki, Y.; Song, K. W.; Caruge, J.-M.; Kazlas, P. T.; Coe-Sullivan, S.; Andrew, T. L.; Bawendi, M. G.; Bulović, V. Qleds for Displays and Solid-State Lighting. MRS Bull. 2013, 38, 703-711.

(6) Gaponenko, S.; Demir, H. V.; Seassal, C.; Woggon, U. Colloidal Nanophotonics: The Emerging Technology Platform. Opt. Express 2016, 24, A430-A433.

(7) Klimov, V. I.; Mikhailovsky, A. A.; Xu, S.; Malko, A.; Hollingsworth, J. A.; Leatherdale, C. A.; Eisler, H.-J.; Bawendi, M. G. Optical Gain and Stimulated Emission in Nanocrystal Quantum Dots. Science 2000, 290, 314-317.

(8) Asada, M.; Miyamoto, Y.; Suematsu, Y. Gain and the Threshold of Three-Dimensional Quantum-Box Lasers. IEEE J. Quantum Electron. 1986, 22, 1915-1921.

(9) Klimov, V. I.; Mikhailovsky, A. A.; McBranch, D. W.; Leatherdale, C. A.; Bawendi, M. G. Quantization of Multiparticle Auger Rates in Semiconductor Quantum Dots. Science 2000, 287, 1011-1013.

(10) Klimov, V. I.; Ivanov, S. A.; Nanda, J.; Achermann, M.; Bezel, I.; McGuire, J. A.; Piryatinski, A. Single-Exciton Optical Gain in Semiconductor Nanocrystals. Nature 2007, 447, 441-446.

(11) Klimov, V. I. Spectral and Dynamical Properties of Multiexcitons in Semiconductor Nanocrystals. Annu. Rev. Phys. Chem. 2007, 58, 635673.

(12) Dang, C.; Lee, J.; Breen, C.; Steckel, J. S.; Coe-Sullivan, S.; Nurmikko, A. Red, Green and Blue Lasing Enabled by Single-Exciton Gain in Colloidal Quantum Dot Films. Nat. Nanotechnol. 2012, 7, 335339.

(13) Guzelturk, B.; Kelestemur, Y.; Gungor, K.; Yeltik, A.; Akgul, M. Z.; Wang, Y.; Chen, R.; Dang, C.; Sun, H.; Demir, H. V. Stable and LowThreshold Optical Gain in Cdse/Cds Quantum Dots: An All-Colloidal Frequency up-Converted Laser. Adv. Mater. 2015, 27, 2741-2746.

(14) Gao, Y.; Ta, V. D.; Zhao, X.; Wang, Y.; Chen, R.; Mutlugun, E.; Fong, K. E.; Tan, S. T.; Dang, C.; Sun, X. W.; et al. Observation of Polarized Gain from Aligned Colloidal Nanorods. Nanoscale 2015, 7, 6481-6486. 
(15) Kazes, M.; Lewis, D. Y.; Ebenstein, Y.; Mokari, T.; Banin, U. Lasing from Semiconductor Quantum Rods in a Cylindrical Microcavity. Adv. Mater. 2002, 14, 317-321.

(16) Gao, Y.; Yu, G.; Wang, Y.; Dang, C.; Sum, T. C.; Sun, H.; Demir, H. V. Green Stimulated Emission Boosted by Nonradiative Resonant Energy Transfer from Blue Quantum Dots. J. Phys. Chem. Lett. 2016, 7, $2772-2778$.

(17) Roh, K.; Dang, C.; Lee, J.; Chen, S.; Steckel, J. S.; Coe-Sullivan, S.; Nurmikko, A. Surface-Emitting Red, Green, and Blue Colloidal Quantum Dot Distributed Feedback Lasers. Opt. Express 2014, 22, 18800-18806.

(18) Guilhabert, B.; Foucher, C.; Haughey, A. M.; Mutlugun, E.; Gao, Y.; Herrnsdorf, J.; Sun, H. D.; Demir, H. V.; Dawson, M. D.; Laurand, N. Nanosecond Colloidal Quantum Dot Lasers for Sensing. Opt. Express 2014, 22, 7308-7319.

(19) Dang, C.; Lee, J.; Roh, K.; Kim, H.; Ahn, S.; Jeon, H.; Breen, C.; Steckel, J. S.; Coe-Sullivan, S.; Nurmikko, A. Highly Efficient, Spatially Coherent Distributed Feedback Lasers from Dense Colloidal Quantum Dot Films. Appl. Phys. Lett. 2013, 103, 171104.

(20) Zavelani-Rossi, M.; Krahne, R.; Della Valle, G.; Longhi, S.; Franchini, I. R.; Girardo, S.; Scotognella, F.; Pisignano, D.; Manna, L.; Lanzani, G.; et al. Self-Assembled Cdse/Cds Nanorod Micro-Lasers Fabricated from Solution by Capillary Jet Deposition. Laser Photonics Rev. 2012, 6, 678-683.

(21) Nurmikko, A. What Future for Quantum Dot-Based Light Emitters? Nat. Nanotechnol. 2015, 10, 1001-1004.

(22) Erdogan, T.; Hall, D. G. Circularly Symmetric Distributed Feedback Semiconductor Laser: An Analysis. J. Appl. Phys. 1990, 68, 1435-1444.

(23) Erdogan, T.; King, O.; Wicks, G. W.; Hall, D. G.; Anderson, E. H.; Rooks, M. J. Circularly Symmetric Operation of a Concentric-CircleGrating, Surface- Emitting, Algaas/Gaas Quantum-Well Semiconductor Laser. Appl. Phys. Lett. 1992, 60, 1921-1923.

(24) Erdogan, T.; Hall, D. G. Circularly Symmetric Distributed Feedback Laser: Coupled Mode Treatment of Te Vector Fields. IEEE J. Quantum Electron. 1992, 28, 612-623.

(25) Chen, Y.; Guilhabert, B.; Herrnsdorf, J.; Zhang, Y.; Mackintosh, A. R.; Pethrick, R. A.; Gu, E.; Laurand, N.; Dawson, M. D. Flexible Distributed-Feedback Colloidal Quantum Dot Laser. Appl. Phys. Lett. 2011, 99, 241103.

(26) Chen, Y.; Li, Z.; Zhang, Z.; Psaltis, D.; Scherer, A. Nanoimprinted Circular Grating Distributed Feedback Dye Laser. Appl. Phys. Lett. 2007, 91, 051109.

(27) Bae, W. K.; Kwak, J.; Lim, J.; Lee, D.; Nam, M. K.; Char, K.; Lee, C.; Lee, S. Multicolored Light-Emitting Diodes Based on All-QuantumDot Multilayer Films Using Layer-by-Layer Assembly Method. Nano Lett. 2010, 10, 2368-2373.

(28) Wang, Y.; Ta, V. D.; Gao, Y.; He, T. C.; Chen, R.; Mutlugun, E.; Demir, H. V.; Sun, H. D. Stimulated Emission and Lasing from Cdse/ Cds/Zns Core-Multi-Shell Quantum Dots by Simultaneous ThreePhoton Absorption. Adv. Mater. 2014, 26, 2954-2961.

(29) Cragg, G. E.; Efros, A. L. Suppression of Auger Processes in Confined Structures. Nano Lett. 2010, 10, 313-317.

(30) Herrnsdorf, J.; Guilhabert, B.; Chen, Y.; Kanibolotsky, A. L.; Mackintosh, A. R.; Pethrick, R. A.; Skabara, P. J.; Gu, E.; Laurand, N.; Dawson, M. D. Flexible Blue-Emitting Encapsulated Organic Semiconductor Dfb Laser. Opt. Express 2010, 18, 25535-25545.

(31) Joannopoulos, J. D.; Johnson, S. G.; Winn, J. N.; Meade, R. D. Photonic Crystals: Molding the Flow of Light, 2nd ed.; Princeton University Press, 2011.

(32) Kopp, V. I.; Fan, B.; Vithana, H. K. M.; Genack, A. Z. LowThreshold Lasing at the Edge of a Photonic Stop Band in Cholesteric Liquid Crystals. Opt. Lett. 1998, 23, 1707-1709.

(33) Bauer, C.; Giessen, H.; Schnabel, B.; Kley, E. B.; Schmitt, C.; Scherf, U.; Mahrt, R. A Surface-Emitting Circular Grating Polymer Laser. Adv. Mater. 2001, 13, 1161-1164.

(34) Jordan, R. H.; Hall, D. G.; King, O.; Wicks, G.; Rishton, S. Lasing Behavior of Circular Grating Surface-Emitting Semiconductor Lasers. J. Opt. Soc. Am. B 1997, 14, 449-453. 